\title{
Production of Cellulases by Solid State Fermentation of Different Agricultural Residues Using Humicola insolens MTCC 1433
}

\author{
Diksha Singla $^{1^{*}}$ and Monica Sachdeva Taggar ${ }^{2}$ \\ ${ }^{1}$ Department of Biochemistry, Punjab Agricultural University, Ludhiana-141004, Punjab, India \\ ${ }^{2}$ School of Renewable Energy Engineering, Punjab Agricultural University, \\ Ludhiana-141004, Punjab, India \\ *Corresponding author
}

\section{A B S T R A C T}

\section{Keywords}

Agricultural residues, Solid state

fermentation, FPase,

CMCase, Cellobiase,

Xylanase.

\section{Article Info}

Accepted:

12 September 2017

Available Online:

10 November 2017
The production of cellulases is an important strategy for the development of sustainable second-generation ethanol production processes. The present study was undertaken to select cheap and efficient agricultural residue suitable for cellulase production using fungus Humicola insolens. Six different agricultural residues viz., paddy straw, soybean pod husk, sugarcane bagasse, groundnut shells, corn stalks and pigeonpea pod husk were used as substrate in the solid state fermentation medium. Among all the substrates, maximum filter paper (FPase), carboxymethyl cellulase (CMCase), cellobiase and xylanase activities of $71.28 \mathrm{nmol} / \mathrm{min} / \mathrm{gds}, 227.33 \mathrm{nmol} / \mathrm{min} / \mathrm{gds}, 101.02 \mathrm{nmol} / \mathrm{min} / \mathrm{gds}$, $1988.62 \mathrm{nmol} / \mathrm{min} / \mathrm{gds}$ respectively were recorded at 72 hours after incubation using soybean pod husk as substrate, followed by sugarcane bagasse with $58.78 \mathrm{nmol} / \mathrm{min} / \mathrm{gds}$ FPase, $225.55 \mathrm{nmol} / \mathrm{min} / \mathrm{gds}$ CMCase, $96.10 \mathrm{nmol} / \mathrm{min} / \mathrm{gds}$ cellobiase and 1727.94 $\mathrm{nmol} / \mathrm{min} / \mathrm{gds}$ xylanase activities. Soybean pod husk showed maximum cellulase activity as compared to all comparatively other residues.

\section{Introduction}

The existence of pollution problems associated with agricultural wastes, scarcity of places for their disposal, costlier treatment options and increased need to save valuable resources have necessitated the utilization and bioconversion of waste into high value industrially useful products. Presently, huge amounts of agricultural and industrial cellulosic wastes have been accumulating in environment which can be considered as "waste" and can potentially be used to produce various value added products like enzymes, biofuels, animal feeds, chemicals, etc. (Abo-state et al., 2013). Cellulose has attracted worldwide attention as a renewable resource that can be converted into bio-based products and bioenergy ( $\mathrm{Li}$ et al., 2009). Cellulose is a crystalline and linear polymer of thousands of D-glucose residues linked by $\beta$-1, 4-glycosidic bonds, and is considered the most abundant and renewable biomass resource and a formidable reserve of raw material (Quiroz-castaneda and Folch-mallol, 2013). Cellulose present in the lignocellulosic biomass is considered as one of the most important reservoirs of carbon for the production of glucose, a fuel and chemical feedstock (Kalogeris et al., 2003). The most 
promising technology to produce ethanol from agricultural biomass is based on enzymatic hydrolysis of cellulose by cellulases (Ahamed and Vermette, 2008).

Cellulose is commonly degraded by an enzyme called cellulase. Cellulases are inducible enzymes produced chiefly by fungi, bacteria and protozoan that catalyze the hydrolysis of cellulose (Mahalakshmi and Jayalakshmi, 2016). These microorganisms can be aerobic, anaerobic, mesophilic or thermophilic (Khatiwada et al., 2016). Cellulase is an important and essential enzyme used for catalyzing the depolymerization of cellulose into fermentable sugar (Li et al., 2009).

Among different microbes, fungi are the most studied group of cellulose-degrading microorganisms, owing to their high protein secretion capabilities, multi-component and synergetic cellulolytic enzyme activities (Obeng et al., 2017). Fungal cellulases have the potential to digest cellulose, hemicelluloses and lignin by secreting diverse set of hydrolytic and oxidative enzymes (Fatma et al., 2010). The most accepted commercial microbes that are found to be highly cellulolytic are Aspergillus fumigatus, A. nidulans, $A$. acculeatus, $A$. niger, $A$. oryzae (recombinant), Melanocorpus albomyces, Trichoderma koningii, T. viride, Penicillium fumiculosum, Talaromyces emersonii, Humicola grisea, H. insolens, Fusarium solani, Irpex lactius, Sclerotium rolfsii, etc. (Imran et al., 2016).

The bioconversion of cellulose to fermentable sugars requires the synergistic action of complete cellulase system comprising of 1, 4$\beta$-D glucanglucanohydrolase (EC 3.2.1.3), 1, $4-\beta-D \quad$ glucancellobiohydrolyase (EC 3.2.1.91) and $\beta$-D glucosidase (EC 3.2.1.21). These enzymes are commonly referred to as endoglucanase, exoglucanase and cellobiase, respectively. The endoglucanases attack randomly and cleave the cellulose chains to form glucose, cellobiose and cellotriose. The exoglucanases attack the non-reducing end of cellulose to form the cellobiose units. Finally, cellobiase converts cellobiose into D-glucose (Gupta and Verma, 2015). In addition to the three major groups of cellulase enzymes, there is also the hemicellulase system which involves xylanase (endo-1, 4- D-xylan xylanohydrolase, EC 3.2.1.8) that catalyzes the hydrolysis of xylan to produce a mixture of shorter xylo-oligosaccharides, xylose and xylobiose (Abo-state et al., 2013).

The production of cellulases is a major cost factor for the nascent lignocellulosic biofuel industry. The operational cost of enzymes results from two main factors, i.e. quality of enzyme required to hydrolyze biomass and cost of each kilogram of enzyme to the end user (Ellila et al., 2017). Utilization of agricultural residues as substrates can help to reduce production cost of microbial enzymes and in addition, help in the management of various agricultural residues which are generated in abundance and difficult to dispose-off (Bajaj et al., 2014).

For understanding the potential of different plant-based agricultural residues available in abundance, it is necessary to develop a lowcost growth medium for fungal strain with high cellulase production. Therefore, the present study was carried out to study fungal cellulase production using different agricultural residues.

\section{Materials and Methods}

\section{Sources of agricultural waste}

The substrates used for this study included paddy straw, soybean pod husk, sugarcane bagasse, groundnut shells, corn stalks and pigeonpea pod husk. Agricultural residues 
were procured from Department of Plant Breeding and Genetics, Punjab Agricultural University, Ludhiana. The residues were thoroughly washed with tap water to remove soil, dust and other unwanted materials prior to sun drying. The residues were, then, milled using an electric grinder and sieved through 30 mesh screen. The powdered residues were stored in polythene bags at room temperature for subsequent studies.

\section{Preparation of inoculum}

The fungus Humicola insolens (MTCC 1433) was procured from Microbial Type Culture Collection (MTCC), Institute of Microbial Technology (IMTECH), Chandigarh and maintained on Yeast powder-soluble starch (YPSS) agar slants at $4^{\circ} \mathrm{C}$. The culture was inoculated on YPSS medium at $45^{\circ} \mathrm{C}$ for 5-7 days of incubation period. After the incubation period, the spores were obtained by rinsing the plates with $10 \mathrm{ml}$ sterile saline water $(0.9 \%)$ and the spores were collected in a sterile tube. The spore count was determined with a haemocytometer and the spore concentration of $1 \times 10^{8}$ spores $/ \mathrm{ml}$ was used as inoculum for cellulase production.

\section{Cellulase production by solid state fermentation process}

In order to select the best substrate for the enzyme production, experiments were carried out in $250 \mathrm{ml}$ Erlenmeyer flasks containing $2.5 \mathrm{~g}$ each of the substrate of 30 mesh particle size and moistened with Mandels medium in a ratio of 1:4. Mandels and Weber medium (1 litre) contained $2 \mathrm{~g} \quad \mathrm{KH}_{2} \mathrm{PO}_{4}, \quad 1.4 \mathrm{~g}$ $\left(\mathrm{NH}_{4}\right)_{2} \mathrm{SO}_{4}, 1 \mathrm{~g}$ peptone, $0.3 \mathrm{~g} \mathrm{MgSO}_{4} \cdot 7 \mathrm{H}_{2} \mathrm{O}$, $0.3 \mathrm{~g} \mathrm{CaCl}_{2}, 5 \mathrm{mg} \mathrm{FeSO}_{4} .7 \mathrm{H}_{2} \mathrm{O}, 1.56 \mathrm{mg}$ $\mathrm{MnSO}_{4} \cdot \mathrm{H}_{2} \mathrm{O}, 1.4 \mathrm{mg} \mathrm{ZnSO}_{4} .7 \mathrm{H}_{2} \mathrm{O}, 2.0 \mathrm{mg}$ $\mathrm{CoCl}_{2} \cdot 6 \mathrm{H}_{2} \mathrm{O}$ and $1 \mathrm{ml}$ Tween 80 (Mandels and Weber, 1969). The $\mathrm{pH}$ of the medium was adjusted to 5.0. The residues were, then, subjected to steam pre-treatment by autoclaving at $121^{\circ} \mathrm{C}(15 \mathrm{psi})$ for 20 minutes and cooled before inoculation. Following the autoclaving of the flasks, $1 \mathrm{ml}$ of fungal spore suspension per gram of residue was inoculated into different flasks and incubated at $45^{\circ} \mathrm{C}$ for $168 \mathrm{~h}$.

\section{Enzyme extraction}

Enzyme extraction was carried out at an interval of $24 \mathrm{~h}$ for a period of 7 days. For this, $30 \mathrm{ml}$ of $0.1 \mathrm{M}$ chilled citrate buffer $(\mathrm{pH}$ 4.8) was added to each flask and the flasks were then incubated at $27^{\circ} \mathrm{C}$ in an orbital shaking incubator with a shaking speed of 100 rpm for $10 \mathrm{~min}$. The contents were subsequently filtered through Whatman filter paper No. 1 followed by centrifugation at $10,000 \mathrm{rpm}$ at $4^{\circ} \mathrm{C}$ for $15 \mathrm{~min}$. The supernatant, thus, obtained was dialyzed against same buffer (10X diluted) and assayed for different cellulolytic enzyme activities.

\section{Determination of enzymatic activity}

The enzymatic extract was used to determinate filter paper cellulase, carboxymethyl cellulase (CMCase), cellobiase and xylanase activities.

\section{Filter paper cellulase (FPase) activity}

FPase activity of enzyme filtrate was assessed by the method of Mandels et al., (1976). FPase was determined using Whatman no.1 filter paper as substrate. Filter paper strip $(1 \mathrm{~cm} \times 6 \mathrm{~cm})$ was added to a reaction mixture that contained $0.5 \mathrm{~mL} 100 \mathrm{mM}$ citrate buffer $(\mathrm{pH} 4.8)$ and $0.5 \mathrm{~mL}$ enzyme preparation and incubated the mixture at $50^{\circ} \mathrm{C}$ for $60 \mathrm{~min}$. After incubation, the amount of reducing sugars released was measured as per Nelson (1944). Control tubes were run simultaneously by using heat inactivated enzyme. The enzyme activity was expressed as nanomoles of the reducing sugars released 
per min per g dry substrate (nmol/min/gds). The protein content was determined by method of Lowry et al., (1951) and specific activities were expressed as nanomoles of the reducing sugars released per min per $\mathrm{mg}$ protein (nmol/min/mg protein).

\section{Carboxymethyl cellulase (CMCase activity)}

CMCase activity was determined by the method of Wood and Bhat (1988) in a total reaction volume of $1 \mathrm{ml}$ containing $0.3 \mathrm{ml}$ enzyme preparation and $0.5 \mathrm{ml} 0.5 \%(\mathrm{w} / \mathrm{v})$ medium viscosity carboxymethylcellulose solution in citrate buffer $(50 \mathrm{mM}, \mathrm{pH} 4.8)$. This mixture was incubated at $50^{\circ} \mathrm{C}$ for 30 min. The amount of reducing sugars released was measured as per Nelson (1944). The enzyme activity was expressed as nanomoles of the reducing sugars released per min per $\mathrm{g}$ dry substrate (nmol/min/gds) and specific activity was expressed as nanomoles of the reducing sugars released per min per $\mathrm{mg}$ protein (nmol/min/mg protein).

\section{Cellobiase activity}

Cellobiase activity was measured according to the method of Toyama and Ogawa (1977). Cellobiase activity was determined in a total reaction volume of $1 \mathrm{ml}$ containing $0.5 \mathrm{ml}$ enzyme preparation and $0.5 \mathrm{ml} 0.05 \%(\mathrm{w} / \mathrm{v})$ cellobiose solution in citrate buffer $(100 \mathrm{mM}$, $\mathrm{pH}$ 4.8). This mixture was incubated at $50^{\circ} \mathrm{C}$ for $120 \mathrm{~min}$. The amount of reducing sugars was measured as per Nelson (1944). The enzyme activity was expressed as nanomoles of the reducing sugars released per min per $\mathrm{g}$ dry substrate (nmol/min/gds) and specific activity was expressed as nanomoles of the reducing sugars released per min per $\mathrm{mg}$ protein (nmol/min/mg protein).

\section{Xylanase activity}

Xylanase activity was determined as described by Bailey et al (1992). For determination of xylanase activity, $0.5 \mathrm{ml}$ of substrate $(1 \%$, w/v, xylan) in citrate buffer was incubated at $50^{\circ} \mathrm{C}$ with $0.5 \mathrm{ml}$ enzyme preparation for $10 \mathrm{~min}$, and the hydrolysis product was measured by Nelson (1944). The enzyme activity was expressed as nanomoles of the xylose released per min per g dry substrate (nmol/min/gds) and specific activity was expressed as nanomoles of the xylose released per min per $\mathrm{mg}$ protein (nmol/min/mg protein).

\section{Statistical analysis}

The data pertaining to enzyme activities of different plant based agricultural residues using $H$. insolens were analysed using analysis of variance (ANOVA) to test the differences among treatments in factorial CRD using CPCS1 software.

\section{Results and Discussion}

In the present study, six agricultural residues, viz. paddy straw, soybean pod husk, sugarcane bagasse, groundnut shells, corn stalks and pigeonpea pod husk were used as substrate under solid state fermentation for cellulase production by fungi Humicola insolens. The filter paper, CMCase, cellobiase and xylanase activities on different substrates were determined and discussed as under.

The filter paper activity of $H$. insolens (MTCC 1433) using different agricultural residues as a substrate was studied and the results of the same have been presented in Table 1. Significantly highest mean filter paper activity of $46.40 \mathrm{nmol} / \mathrm{min} / \mathrm{gds}$ was observed using soybean pod husk as a substrate under solid state fermentation. Significantly highest mean filter paper activity of $46.39 \mathrm{nmol} / \mathrm{min} / \mathrm{gds}$ was observed at 72 hours after incubation, followed by mean activity of $37.11 \mathrm{nmol} / \mathrm{min} / \mathrm{gds}$ at 96 hours after incubation. The interaction between different incubation times and 
agricultural residues as substrates revealed that there were significant differences in the filter paper activity of $H$. insolens. Significantly highest filter paper activity of $71.28 \mathrm{nmol} / \mathrm{min} / \mathrm{gds}$ was observed using soybean pod husk as substrate at 72 hours after incubation, followed by filter paper activity of $58.78 \mathrm{nmol} / \mathrm{min} / \mathrm{gds}$ with sugarcane bagasse as substrate. Similarly, highest specific activity was observed in soybean pod husk as represented in parentheses in Table 1.

The mean CMCase activity of $H$. insolens (Table 2) using different agricultural residues varied significantly. Significantly highest mean CMCase activity of 149.91 $\mathrm{nmol} / \mathrm{min} / \mathrm{gds}$ was observed using soybean pod husk as a substrate under solid state fermentation. Significantly highest mean CMCase activity of $148.07 \mathrm{nmol} / \mathrm{min} / \mathrm{gds}$ was observed at 72 hours after incubation, followed by mean activity of 122.43 $\mathrm{nmol} / \mathrm{min} / \mathrm{gds}$ at 96 hours after incubation. The interaction between different incubation times and agricultural residues as substrates was also significant and revealed that there were significant differences in the CMCase activity of $H$. insolens. Significantly highest CMCase activity of $227.33 \mathrm{nmol} / \mathrm{min} / \mathrm{gds}$ was observed using soybean pod husk as substrate at 72 hours after incubation. Similarly, highest specific activity was observed in soybean pod husk as depicted in Table 2.

The cellobiase activity of $H$. insolens has been presented in Table 3. Significantly highest mean cellobiase activity of 78.09 $\mathrm{nmol} / \mathrm{min} / \mathrm{gds}$ observed using soybean pod husk as a substrate under solid state fermentation. Significantly highest mean cellobiase activity of $73.74 \mathrm{nmol} / \mathrm{min} / \mathrm{gds}$ was observed at 72 hours after incubation, followed by mean activity of 65.17 $\mathrm{nmol} / \mathrm{min} / \mathrm{gds}$ at 96 hours after incubation. The interaction between different incubation times and agricultural residues as substrates revealed that there were significant differences in the cellobiase activity of $H$. insolens. Significantly highest cellobiase activity of $101.02 \mathrm{nmol} / \mathrm{min} / \mathrm{gds}$ was observed using soybean pod husk as substrate at 72 hours after incubation. Similarly, highest specific activity was observed in soybean pod husk as as shown in Table 3.

Different agricultural residues were used as substrate under SSF for xylanase production and the xylanase activity of $H$. insolens (MTCC 1433) has been presented in Table 4. The results revealed that significantly highest mean xylanase activity of 1547.06 $\mathrm{nmol} / \mathrm{min} / \mathrm{gds}$ was observed using soybean pod husk as a substrate under solid state fermentation.

Significantly highest mean xylanase activity of $1418.36 \mathrm{nmol} / \mathrm{min} / \mathrm{gds}$ was observed at 72 hours after incubation, followed by mean activity of $1263.16 \mathrm{nmol} / \mathrm{min} / \mathrm{gds}$ at 96 hours after incubation. The interaction between different incubation times and agricultural residues as substrates revealed that there were significant differences in the xylanase activity of $H$. insolens. Significantly highest xylanase activity of $1988.62 \mathrm{nmol} / \mathrm{min} / \mathrm{gds}$ was observed using soybean pod husk as substrate at 72 hours after incubation. Similarly, highest specific activity was observed in soybean pod husk as represented in parentheses in Table 4.

In the present study, soybean pod husk showed maximum enzyme activity as compared to other residues. Medeiros et al (2008) used different carbon sources including wheat bran, oat spelt xylan, cellulose (avicel), oat bran, banana stem and coffee spent ground as source for production of hemicellulose-degerading enzymes ( $\beta$ xylanase, $\quad \beta$-mannanase and $\alpha$ arabinofuranosidase) from $H$. grisea var. thermoidea. 
Table.1 Filter paper activity (nmol/min/gds) of standard fungus Humicola insolens (MTCC 1433) using different agricultural residues as substrate under solid state fermentation

\begin{tabular}{|c|c|c|c|c|c|c|c|c|}
\hline \multirow{2}{*}{$\begin{array}{l}\text { Agricultural } \\
\text { Residues }\end{array}$} & \multicolumn{8}{|c|}{ Filter paper activity (nmol/min/gds) } \\
\hline & $24 \mathrm{~h}$ & $48 \mathrm{~h}$ & $72 \mathrm{~h}$ & $96 \mathrm{~h}$ & $120 \mathrm{~h}$ & $144 \mathrm{~h}$ & $168 \mathrm{~h}$ & Mean \\
\hline \multirow[t]{2}{*}{ Paddy straw } & 11.33 & 22.02 & 46.82 & 41.72 & 21.51 & 13.31 & 6.02 & 23.25 \\
\hline & $(0.76)$ & $(1.47)$ & $(2.82)$ & $(2.30)$ & $(1.32)$ & $(0.81)$ & $(0.39)$ & (1.41) \\
\hline \multirow[t]{2}{*}{ Soybean pod husk } & 37.77 & 47.13 & 71.28 & 52.24 & 46.41 & 39.44 & 30.51 & 46.40 \\
\hline & (10.48) & $(12.29)$ & (17.40) & $(12.37)$ & (10.50) & $(8.33)$ & $(5.88)$ & (11.04) \\
\hline \multirow[t]{2}{*}{ Sugarcane bagasse } & 16.76 & 30.11 & 58.78 & 50.16 & 42.71 & 32.63 & 27.09 & 36.89 \\
\hline & $(4.48)$ & $(7.30)$ & (12.59) & $(10.06)$ & $(7.90)$ & $(5.54)$ & $(4.85)$ & $(7.53)$ \\
\hline \multirow[t]{2}{*}{ Groundnut shells } & 35.38 & 39.81 & 54.70 & 39.81 & 30.96 & 27.56 & 23.13 & 35.91 \\
\hline & $(7.71)$ & $(8.34)$ & (10.41) & (7.94) & $(5.78)$ & $(4.52)$ & $(3.49)$ & $(6.88)$ \\
\hline \multirow[t]{2}{*}{ Corn stalks } & 9.53 & 13.96 & 27.22 & 24.29 & 19.05 & 13.14 & 9.53 & 16.67 \\
\hline & $(0.57)$ & $(0.83)$ & $(1.49)$ & $(1.28)$ & $(1.00)$ & $(0.69)$ & $(0.47)$ & $(0.91)$ \\
\hline \multirow[t]{2}{*}{ Pigeonpea pod husk } & 7.06 & 9.98 & 19.57 & 14.45 & 11.13 & 7.04 & 3.91 & 10.45 \\
\hline & $(0.49)$ & $(0.71)$ & $(1.28)$ & $(0.92)$ & $(0.63)$ & $(0.37)$ & $(0.21)$ & $(0.66)$ \\
\hline \multirow[t]{2}{*}{ Mean } & 19.64 & 27.17 & 46.39 & 37.11 & 28.63 & 22.19 & 16.70 & \\
\hline & $(4.08)$ & $(5.16)$ & (7.66) & (5.81) & $(4.52)$ & (3.38) & $(2.55)$ & \\
\hline \multirow[t]{3}{*}{$C D(p=0.05)$} & \multicolumn{2}{|c|}{ Residues (R) } & \multicolumn{2}{|c|}{ : $0.63(0.11)$} & & & & \\
\hline & \multicolumn{2}{|c|}{ Incubation time (h) } & \multicolumn{2}{|c|}{ : $0.68(0.11)$} & & & & \\
\hline & \multicolumn{2}{|l|}{$\mathbf{R} \times \mathbf{h}$} & \multicolumn{2}{|c|}{ : $1.66(0.28)$} & & & & \\
\hline
\end{tabular}

Values in parentheses are specific activities which are expressed as $\mathrm{nmol} / \mathrm{min} / \mathrm{mg}$ protein

Table.2 CMCase activity (nmol/min/gds) of standard fungus Humicola insolens MTCC 1433 using different agricultural residues as substrate under solid state fermentation

\begin{tabular}{|c|c|c|c|c|c|c|c|c|}
\hline \multirow{2}{*}{$\begin{array}{l}\text { Agricultural } \\
\text { residues }\end{array}$} & \multicolumn{8}{|c|}{ CMCase activity (nmol/min/gds) } \\
\hline & $24 \mathrm{~h}$ & $48 \mathrm{~h}$ & $72 \mathrm{~h}$ & $96 \mathrm{~h}$ & $120 \mathrm{~h}$ & $144 \mathrm{~h}$ & $168 \mathrm{~h}$ & Mean \\
\hline Paddy straw & $\begin{array}{l}33.15 \\
(2.22)\end{array}$ & $\begin{array}{l}67.67 \\
(4.53)\end{array}$ & $\begin{array}{l}111.61 \\
(6.72)\end{array}$ & $\begin{array}{l}98.90 \\
(5.45)\end{array}$ & $\begin{array}{l}84.66 \\
(5.21)\end{array}$ & $\begin{array}{l}76.80 \\
(4.69)\end{array}$ & $\begin{array}{l}37.30 \\
(2.42)\end{array}$ & $\begin{array}{l}72.87 \\
(4.46)\end{array}$ \\
\hline Soybean pod husk & $\begin{array}{l}108.16 \\
(30.02)\end{array}$ & $\begin{array}{l}141.92 \\
(37.01)\end{array}$ & $\begin{array}{l}227.33 \\
(55.51)\end{array}$ & $\begin{array}{l}179.02 \\
(42.40)\end{array}$ & $\begin{array}{l}158.77 \\
(35.93)\end{array}$ & $\begin{array}{l}143.32 \\
(30.26)\end{array}$ & $\begin{array}{c}90.81 \\
(17.50)\end{array}$ & $\begin{array}{l}149.91 \\
(35.52)\end{array}$ \\
\hline Sugarcane bagasse & $\begin{array}{c}65.20 \\
(17.41)\end{array}$ & $\begin{array}{l}101.29 \\
(24.56)\end{array}$ & $\begin{array}{l}225.55 \\
(48.30)\end{array}$ & $\begin{array}{l}188.85 \\
(37.87)\end{array}$ & $\begin{array}{l}130.95 \\
(24.22)\end{array}$ & $\begin{array}{l}106.03 \\
(17.99)\end{array}$ & $\begin{array}{c}71.93 \\
(12.88)\end{array}$ & $\begin{array}{l}127.11 \\
(26.18)\end{array}$ \\
\hline Groundnut shells & $\begin{array}{c}76.37 \\
(16.68)\end{array}$ & $\begin{array}{c}97.29 \\
(20.39)\end{array}$ & $\begin{array}{l}144.21 \\
(27.44)\end{array}$ & $\begin{array}{l}117.49 \\
(23.43)\end{array}$ & $\begin{array}{l}100.05 \\
(18.66)\end{array}$ & $\begin{array}{c}87.38 \\
(14.32)\end{array}$ & $\begin{array}{l}59.67 \\
(8.99)\end{array}$ & $\begin{array}{c}97.49 \\
(18.56)\end{array}$ \\
\hline Corn stalks & $\begin{array}{l}31.47 \\
(1.87)\end{array}$ & $\begin{array}{l}51.39 \\
(3.06)\end{array}$ & $\begin{array}{l}110.45 \\
(6.05)\end{array}$ & $\begin{array}{l}99.46 \\
(5.24)\end{array}$ & $\begin{array}{l}84.70 \\
(4.45)\end{array}$ & $\begin{array}{l}72.38 \\
(3.80)\end{array}$ & $\begin{array}{l}48.07 \\
(2.39)\end{array}$ & $\begin{array}{l}86.50 \\
(3.84)\end{array}$ \\
\hline Pigeonpea pod husk & $\begin{array}{l}17.13 \\
(1.18)\end{array}$ & $\begin{array}{l}29.30 \\
(2.07)\end{array}$ & $\begin{array}{l}69.24 \\
(4.52)\end{array}$ & $\begin{array}{l}50.83 \\
(3.22)\end{array}$ & $\begin{array}{l}38.99 \\
(2.21)\end{array}$ & $\begin{array}{l}21.31 \\
(1.13)\end{array}$ & $\begin{array}{l}11.80 \\
(0.63)\end{array}$ & $\begin{array}{l}34.09 \\
(2.14)\end{array}$ \\
\hline Mean & $\begin{array}{c}55.25 \\
(11.56)\end{array}$ & $\begin{array}{c}81.48 \\
(15.27)\end{array}$ & $\begin{array}{l}148.07 \\
(24.76)\end{array}$ & $\begin{array}{l}122.43 \\
(19.60)\end{array}$ & $\begin{array}{c}99.69 \\
(15.11)\end{array}$ & $\begin{array}{c}84.54 \\
(12.03)\end{array}$ & $\begin{array}{l}53.26 \\
(7.47)\end{array}$ & \\
\hline $\mathrm{CD}(\mathrm{p}=\mathbf{0 . 0 5})$ & $\begin{array}{l}\text { Residues } \\
\text { Incubatior } \\
\mathbf{R} \times \mathbf{h}\end{array}$ & ime (h) & $\begin{array}{l}: 2.09 \\
: 2.26 \\
: 5.53\end{array}$ & & & & & \\
\hline
\end{tabular}

Values in parentheses are specific activities which are expressed as $\mathrm{nmol} / \mathrm{min} / \mathrm{mg}$ protein 
Table.3 Cellobiase activity (nmol/min/gds) of Humicola insolens MTCC 1433 using different agricultural residues as substrate under solid state fermentation

\begin{tabular}{|c|c|c|c|c|c|c|c|c|}
\hline \multirow[t]{2}{*}{ Agricultural residues } & \multicolumn{8}{|c|}{ Cellobiase activity (nmol/min/g-ds) } \\
\hline & $24 \mathrm{~h}$ & $48 \mathrm{~h}$ & $72 \mathrm{~h}$ & $96 \mathrm{~h}$ & $120 \mathrm{~h}$ & $144 \mathrm{~h}$ & $168 \mathrm{~h}$ & Mean \\
\hline \multirow[t]{2}{*}{ Paddy straw } & 37.21 & 41.58 & 60.61 & 57.41 & 41.87 & 21.60 & 13.15 & 39.06 \\
\hline & $(2.49)$ & $(2.78)$ & $(3.65)$ & $(3.16)$ & $(2.58)$ & $(1.32)$ & $(0.85)$ & $(2.41)$ \\
\hline \multirow[t]{2}{*}{ Soybean pod husk } & 56.31 & 74.18 & 101.02 & 89.69 & 83.08 & 74.37 & 67.95 & 78.09 \\
\hline & (15.63) & (19.34) & $(24.66)$ & $(21.24)$ & (18.80) & (15.71) & (13.09) & (18.35) \\
\hline \multirow[t]{2}{*}{ Sugarcane bagasse } & 49.69 & 63.37 & 96.10 & 77.88 & 59.88 & 53.54 & 44.27 & 63.53 \\
\hline & $(13.27)$ & $(15.36)$ & $(20.58)$ & $(15.62)$ & $(11.07)$ & $(9.09)$ & $(7.93)$ & (13.27) \\
\hline \multirow[t]{2}{*}{ Groundnut shells } & 46.02 & 57.76 & 83.20 & 73.67 & 58.19 & 50.77 & 38.02 & 58.23 \\
\hline & (10.04) & $(12.11)$ & $(15.83)$ & $(14.70)$ & $(10.85)$ & $(8.32)$ & $(5.73)$ & (11.08) \\
\hline \multirow[t]{2}{*}{ Corn stalks } & 27.05 & 44.52 & 56.31 & 52.10 & 40.15 & 18.81 & 13.47 & 36.06 \\
\hline & (1.61) & $(2.66)$ & (3.09) & $(2.74)$ & $(2.11)$ & $(0.99)$ & $(0.67)$ & (1.98) \\
\hline \multirow[t]{2}{*}{ Pigeonpea pod husk } & 14.54 & 25.74 & 45.17 & 40.25 & 35.46 & 17.74 & 8.84 & 26.82 \\
\hline & $(1.00)$ & (1.82) & $(2.95)$ & $(2.55)$ & $(2.01)$ & $(0.94)$ & $(0.47)$ & (1.68) \\
\hline \multirow[t]{2}{*}{ Mean } & 38.47 & 51.19 & 73.74 & 65.17 & 53.11 & 39.47 & 30.95 & \\
\hline & $(7.34)$ & (9.01) & (11.79) & $(\mathbf{1 0 . 0 0 )}$ & (7.90) & $(6.06)$ & $(4.79)$ & \\
\hline \multirow[t]{3}{*}{$\mathrm{CD}(\mathrm{p}=0.05)$} & \multicolumn{2}{|c|}{ Residues (R) } & \multicolumn{2}{|c|}{$: 0.36(0.09)$} & & & & \\
\hline & \multicolumn{2}{|c|}{ Incubation time (h) } & \multicolumn{2}{|c|}{ : $0.39(0.09)$} & & & & \\
\hline & \multicolumn{2}{|l|}{$\mathbf{R} \times \mathbf{h}$} & \multicolumn{2}{|c|}{ : $0.96(0.23)$} & & & & \\
\hline
\end{tabular}

Values in parentheses are specific activities which are expressed as $\mathrm{nmol} / \mathrm{min} / \mathrm{mg}$ protein

Table.4 Xylanase activity (nmol/min/gds) of standard fungus Humicola insolens MTCC 1433 using different agricultural residues as substrate under solid state fermentation

\begin{tabular}{|c|c|c|c|c|c|c|c|c|}
\hline \multirow{2}{*}{$\begin{array}{l}\text { Agricultural } \\
\text { residues }\end{array}$} & \multicolumn{8}{|c|}{ Xylanase activity (nmol/min/g-ds) } \\
\hline & $24 \mathrm{~h}$ & $48 \mathrm{~h}$ & $72 \mathrm{~h}$ & $96 \mathrm{~h}$ & $120 \mathrm{~h}$ & $144 \mathrm{~h}$ & $168 \mathrm{~h}$ & Mean \\
\hline Paddy $\mathrm{s}$ & $\begin{array}{c}1097.34 \\
(73.41)\end{array}$ & $\begin{array}{l}1251.58 \\
(83.77)\end{array}$ & $\begin{array}{c}1471.73 \\
(88.63)\end{array}$ & $\begin{array}{c}1329.72 \\
(73.27)\end{array}$ & $\begin{array}{c}1165.58 \\
(71.73)\end{array}$ & $\begin{array}{c}1053.64 \\
(64.32)\end{array}$ & $\begin{array}{l}930.79 \\
(60.46)\end{array}$ & $\begin{array}{c}1185.77 \\
(73.66)\end{array}$ \\
\hline Soybean $\mathrm{p}$ & $\begin{array}{l}1246.58 \\
(345.92)\end{array}$ & $\begin{array}{l}1494.46 \\
(389.70)\end{array}$ & $\begin{array}{l}1988.62 \\
(485.47)\end{array}$ & $\begin{array}{r}1747.80 \\
(413.89)\end{array}$ & $\begin{array}{l}1572.38 \\
(355.81)\end{array}$ & $\begin{array}{c}1476.62 \\
(311.84)\end{array}$ & $\begin{array}{l}1302.97 \\
(250.98)\end{array}$ & $\begin{array}{l}1547.06 \\
(364.80)\end{array}$ \\
\hline Sugarca & $\begin{array}{c}949.37 \\
(253.63)\end{array}$ & $\begin{array}{l}1162.24 \\
(281.75)\end{array}$ & $\begin{array}{l}1727.94 \\
(370.01)\end{array}$ & $\begin{array}{c}1625.79 \\
(326.01)\end{array}$ & $\begin{array}{l}1452.86 \\
(268.70)\end{array}$ & $\begin{array}{l}1327.23 \\
(225.25)\end{array}$ & $\begin{array}{l}1189.34 \\
(212.98)\end{array}$ & $\begin{array}{l}1347.83 \\
(276.91)\end{array}$ \\
\hline Groundnut & $\begin{array}{l}1032.08 \\
(225.29)\end{array}$ & $\begin{array}{l}1336.07 \\
(280.15)\end{array}$ & $\begin{array}{l}1649.48 \\
(313.90)\end{array}$ & $\begin{array}{c}1400.72 \\
(279.41)\end{array}$ & $\begin{array}{l}1342.63 \\
(250.39)\end{array}$ & & $\begin{array}{l}1105.07 \\
(166.62)\end{array}$ & $\begin{array}{l}1298.14 \\
(245.13)\end{array}$ \\
\hline Cor & $\begin{array}{l}458.73 \\
(27.30)\end{array}$ & $\begin{array}{l}791.47 \\
(47.26)\end{array}$ & $\begin{array}{c}1282.58 \\
(70.31)\end{array}$ & $\begin{array}{c}1165.08 \\
(61.38)\end{array}$ & $\begin{array}{c}1077.90 \\
(56.62)\end{array}$ & $\begin{array}{l}930.50 \\
(48.88)\end{array}$ & $\begin{array}{l}854.04 \\
(42.44)\end{array}$ & $\begin{array}{l}937.19 \\
(\mathbf{5 0 . 6 0 )}\end{array}$ \\
\hline Piger & $\begin{array}{l}135.06 \\
(9.30)\end{array}$ & $\begin{array}{l}183.86 \\
(13.00)\end{array}$ & $\begin{array}{l}389.81 \\
(25.48)\end{array}$ & $\begin{array}{l}309.84 \\
(19.66)\end{array}$ & $\begin{array}{l}239.75 \\
(13.62)\end{array}$ & $\begin{array}{l}151.51 \\
(8.02)\end{array}$ & $\begin{array}{l}90.55 \\
(4.80)\end{array}$ & $\begin{array}{l}214.34 \\
(13.41)\end{array}$ \\
\hline Mean & $\begin{array}{c}819.86 \\
(155.81)\end{array}$ & $\begin{array}{l}1036.61 \\
(182.61)\end{array}$ & $\begin{array}{l}1418.36 \\
(225.64)\end{array}$ & $\begin{array}{l}1263.16 \\
(195.60)\end{array}$ & $\begin{array}{c}1141.85 \\
(169.48)\end{array}$ & $\begin{array}{r}1026.74 \\
(143.07)\end{array}$ & $\begin{array}{c}912.13 \\
(123.05)\end{array}$ & \\
\hline CD & $\begin{array}{l}\text { Residues } \\
\text { Incubatio } \\
\mathbf{R} \times \mathbf{h}\end{array}$ & time (h) & $\begin{array}{l}: 5.73( \\
: 6.18 \\
: 15.15\end{array}$ & $\begin{array}{l}2.06) \\
2.22) \\
(5.45)\end{array}$ & & & & \\
\hline
\end{tabular}

Values in parentheses are specific activities which are expressed as $\mathrm{nmol} / \mathrm{min} / \mathrm{mg}$ protein 
Coffee spent-ground was selected as the best substrate for the production of hemicellulase activity, followed by wheat bran. Delabona et al., (2012) studied the cellulolytic enzymes production by A. fumigatus using different substrates viz., wheat bran, sugarcane bagasse, soybean bran and orange peel during solid state fermentation (SSF) process. Soybean bran showed maximal endoglucanase production (160.1 IU $\mathrm{g}^{-1}$ of substrate), followed by wheat bran $\left(122.8 \mathrm{IU} \mathrm{g}^{-1}\right)$ and a mixture of sugarcane bagasse and wheat bran $(1: 1) \quad\left(112.6 \quad\right.$ IU $\left.\quad \mathrm{g}^{-1}\right)$. The highest endogluconase production for all three substrates was achieved after $72 \mathrm{~h}$ of incubation. Bajaj et al., (2014) reported that Sporotrichum thermophile LAR5 fungal isolate produced significant amount of cellulase on low-cost agro-residues as substrates. Wheat bran supported maximum cellulase production (2000 IU ${ }^{-1}$ ) followed by maize bran (1800 $\left.\mathrm{IU} \mathrm{l}^{-1}\right)$ and rice husk (1600 $\mathrm{IU}^{-1}$ ). Borkar (2016) studied carboxymethyl cellulase enzyme production by Myriococcum albomyces and $H$. insolens on four different agricultural wastes viz., wheat straw, corn cob, jowar straw and groundnut shell. Humicola insolens showed maximum CMCase activity with corn cob, wheat straw and jowar straw after 4 days of incubation, whereas $M$. albomyces showed maximum activity with corn cob and jowar straw. These fungi were able to degrade agricultural wastes at faster rate by degrading cellulose. Ellila et al., (2017) developed a simple, cost efficient cellulase production process that could be employed locally at a Brazilian sugarcane biorefinery. The low cost industrial residues were used to evaluate their potential for cellulase production and among the solid residues tested, soybean hulls showed the most desirable characteristics.

Cellulase enzyme could be used for conversion of cellulose into fermentable sugars, and cellulase based bio-refinery technologies are versatile and flexible because they utilize cheaper substrates for production of enzyme (Mane et al., 2007). The ability to degrade cellulose is a character distributed among a wide variety of cellulolytic fungi. Studies dealing with cellulase production by fungi using low-cost residues under solid state fermentation are going on throughout the world to enhance the production and purity of fungal cellulases.

In conclusion, based on the results of present studies, soybean pod husk was found to be the most efficient substrate for enzyme production. Soybean pod husk showed higher activities in terms of FPase, CMCase, cellobiase and xylanase after 72 hours of incubation. This study, thus, concludes that soybean pod husk could be used as a potential substrate for production of cellulase and xylanase under solid state fermentation.

\section{Acknowledgment}

The authors thanks the Director, School of Renewable Energy Engineering, P.A.U, Ludhiana for providing necessary facilities to carry out these studies.

\section{References}

Abo-state, M. A. M., M. F. Ghaly and Abdellah E. M. 2013. Production of cellulases and xylanase by thermophilic and alkaliphilic bacterial strains isolated from agricultural wastes. World Appl. Sci. J. 22 (11): 1603-1612.

Ahamed, A., and Vermette, P. 2008. Culturebased strategies to enhance cellulase enzyme production from Trichoderma reesei RUT-C30 in bioreactor culture conditions. Biochem. Eng. J. 40, 399407.

Bailey, M. J., P. Biely and Poutanen K. 1992. Interlaboratory testing of methods for assay of xylanase activity. J. 
Biotechnol. 23, 257-270.

Bajaj, B. K., M. Sharma and Rao R. S. 2014. Agricultural residues for production of cellulase from Sporotrichum thermophile LAR5 and its application for saccharification of rice straw. J. Mater. Environ. Sci. 5(5): 1454-1460.

Borkar, K. M., 2016. Carboxymethyl cellulase activity of thermophilic fungi from different substrates. Int. J. Life Sci. 6, 55-58.

Delabona, P. D. S., R. D. P. B. Pirota, C. A. Codima, C. R. Tremacoldi, A. Rodrigues and Farinas C. S. 2012. Using Amazon forest fungi and agriculture residues as a strategy to produce cellulolytic enzymes. . Biomass Bioenerg. 37, 243-250.

Ellila, S., L. Fonseca, C. Uchima, J. Cota, G. H. Goldman, M. Saloheimo, V. Sacon and Siika-aho M. 2017. Development of a low-cost cellulase production process using Trichoderma reesei for Brazilian biorefineries. Biotechnol. Biofuels. 10, 30-47.

Fatma, H., A. El-Zaher and Fadel M. 2010. Production of bioethanol via enzymatic saccharification of rice straw by cellulase produced by Trichoderma reesei under solid state fermentation. New York Sci. J. 3, 72-78.

Gupta, A., and Verma, J. P. 2015. Sustainable bio-ethanol production from agroresidues: a review. Renew. Sust. Energ. Rev. 41, 550-67.

Imran, M., Z. Anwar, M. Irshad, M. J. Asad and Ashfaq H. 2016. Cellulase production from species of fungi and bacteria from agricultural wastes and its utilization in industry: a review. Adv. Enzyme Res. 4, 44-55.

Kalogeris, E., P. Christakopoulos, P. Katapodis, A. Alexiou, S. Vlachou, D. Kekos and Macris B. J. 2003. Production and characterization of cellulolytic enzymes from the thermophilic fungus Thermoascus aurantiacus under solid state cultivation of agricultural wastes. Process Biochem. 38, 1099-1104.

Khatiwada, P., J. Ahmed, M. H. Sohag, K. Islam and Azad A. K. 2016. Isolation, screening and characterization of cellulase producing bacterial isolates from municipal solid wastes and rice straw wastes. J. Bioprocess. Biotech. 6, $1-5$.

Li, X. H., H. J. Yang, B. Roy, D. Wang, W. F. Yue, L. J. Jiang, E. Y. Park and Miao Y. G. 2009. The most stirring technology in future: Cellulase enzyme and biomass utilization. Afr. J. Biotechnol., 8(11): 2418-2422.

Lowry, O. H., N. J. Rosebrough, A. L. Farr and Randall R. J. 1951. Protein measurement with the Folin phenol reagent. J. Biol. Chem. 193, 265-275.

Mahalakshmi, M., and Jayalakshmi, S. 2016. Cellulase production by Aspergillus niger under solid state fermentation using agro industrial wastes. Int. J. Adv. Multidiscip. Res. 3(2): 78-83.

Mandels, M., and Weber, J. 1969. The production of cellulases. In: Hajny, G. J., and Reese, E. T. (Eds.), Cellulases and Their Applications, American Chemical Society, Washington DC, pp. 391-414.

Mandels, M., R. Andreotti and Roche C. 1976. Measurement of saccharifying cellulase. Biotechnol. Bioeng. Symp., US Army Natick Development Center, Nauck, MA., pp 21-33.

Mane, V. P., S. S. Patil, A. A. Syed and Baig M. M. 2007. Bioconversion of low quality lignocellulosic agricultural waste into edible protein by Pleurotus sajor-caju (Fr.) Singer. J. Zhejiang Univ. Sci. B. 8, 745- 751.

Medeiros, R. G., L. A. Coelho and Filho, E. X. F. 2008. Agricultural residues as source for the production of 
hemicellulases from Humicola grisea var. thermoidea. Dynamic biochemistry, Process Biotechnol. Mol. Biol. 2(1): 3033.

Nelson, N. 1944. A photometric adaptation of the Somogyi method for the determination of glucose. J. Biol. Chem. $153,375-380$.

Obeng, E. M., S. N. N. Adam, C. Budiman, C. M. Ongkudon, R. Maas and Jose J. 2017 Lignocellulases: a review of emerging and developing enzymes, systems, and practices. Bioresour. Bioprocess. 4, 16-38.

Quiroz-castaneda, R. E., and Folch-mallol, J. L. 2013. Hydrolysis of biomass mediated by cellulases for the production of sugars. In: Chandel, A. K. and da Silva, S. S. (Eds.), Sustainable Degradation of Lignocellulosic Biomass-Techniques, Applications and Commercialization. InTech, pp. 119-155.

Saraswati, B., M. R. Kumar, D. J. M. Kumar D.J, P. Balashanmugam, M. D. B Kumaran and Kalaichelvan P. T. 2012. Cellulase production by Bacillus subtilis isolated from Cow Dung. Archives of Applied Science Research. 4 (1): 269279.

Toyama, M., and Ogawa, K. 1977. Cellulase production of Trichoderma viride in solid and submerged culture methods. In: Ghose, T. K. (Ed.), Proc Bioconversion of Cellulosic Substances into Energy, Chemicals and Microbial Protein, Vol. 1, IIT, New Delhi, India, pp. 305-327.

Wood, T. M., and Bhat, K. M. 1988. Methods for measuring cellulase activities. Methods Enzymol. 160, 87-112.

\section{How to cite this article:}

Diksha Singla and Monica Sachdeva Taggar. 2017. Production of Cellulases by Solid State Fermentation of Different Agricultural Residues Using Humicola insolens MTCC 1433. Int.J.Curr.Microbiol.App.Sci. 6(11): 1409-1418. doi: https://doi.org/10.20546/ijcmas.2017.611.168 